\title{
Prediction in General Relativity
}

\author{
C.D. $\mathrm{McCoy}^{*}$
}

1 July 2015

\begin{abstract}
Several authors have claimed that prediction is essentially impossible in the general theory of relativity, the case being particularly strong, it is said, when one fully considers the epistemic predicament of the observer. Each of these claims rests on the support of an underdetermination argument and a particular interpretation of the concept of prediction. I argue that these underdetermination arguments fail and depend on an implausible explication of prediction in the theory. The technical results adduced in these arguments can be related to certain epistemic issues, but can only be misleadingly or mistakenly characterized as related to prediction.
\end{abstract}

\section{Introduction}

Several authors have investigated the subject of prediction in the general theory of relativity (GTR), arguing on the strength of various technical results that making predictions is essentially impossible for observers in the spacetimes permitted by the theory (Geroch, 1977, Hogarth, 1993, 1997, Earman, 1995; Manchak, 2008). On the face of it, such claims seem to be in significant tension with the importance and success of predictions made on the theoretical basis of GTR. The famous experimental predictions of the precession of the perihelion of Mercury and of gravitational lensing, for example, are widely understood as playing a significant role in the confirmation of the theory. Well-known relativist Clifford Will observes as well that, "today, experimental gravitation is a major component of the field, characterized by continuing efforts to test the theory's predictions..." (Will, 2006, 5). If prediction is impossible in GTR, as these authors maintain, just what do physicists using the theory think they are testing and why do they think that its predictions have passed their tests?

That these authors take themselves to be interpreting the concept of prediction in GTR follows from their approach to philosophical questions. To deserve the name "prediction", any theoretical explication thereof should, it seems, bear some important marks of our concept of prediction, and do so in a way that has clear physical significance. The method of the authors mentioned is to consider what information about the spacetime an observer inhabits is available to her directly, and what information can then be deduced about her future on this observational basis. They use these considerations to yield two conditions that must be met in order for an event to be predictable for a given observer; Manchak (2008) adds a novel condition, motivated by "fully considering" the epistemic predicament of the observer. The results proven on the basis of these conditions are understood to represent epistemic limitations on what observers can predict, essentially due to skeptical concerns which arise because of the possibility of underdetermining, observationally indistinguishable spacetimes which foil any given prediction the observer attempts.

${ }^{*}$ Department of Philosophy, University of California San Diego, La Jolla, CA, USA. email: cdmccoy@ucsd. edu 
While there is much to recommend this formalistic method in the philosophy of science and physics in particular, it has obscured crucial epistemological considerations related to predictability, considerations that must be taken in account when considering the interpretation of physical theory. I therefore argue that the interpretation of the mentioned technical results in terms of the concept of prediction is misleading and even outright mistaken. In contrast, I find the generic usage of the term "prediction" in experimental contexts perfectly reasonable from an epistemological point of view.

My argument divides into two. I begin (\$2) by disputing the main two conditions assumed by all the authors mentioned, for they rely on an overly idealized and unphysical conception of observation. These conditions assume that an observer has epistemic access to all and only events in her causal past, but I claim that no physical mechanism can account for such powers (due to the ubiquity of scattering and other physical considerations). This may sound like grist for their mill; if prediction is precluded in the idealized case as their results suggest, it must be all the more problematic in less idealized cases, where obtaining complete sets of observational data requires one to face the physical limitations I discuss as well as ordinary practical difficulties. A realistic conception of the powers of an observer, one that recognizes the need to interpolate and "complete" observational data, suggests that there is not only relatively little epistemic difference between predictions and retrodictions in general relativity, but also no reason to restrict the initial data from which a prediction is determined to an observer's causal past. Indeed, we often make reasonable and successful inductive inferences about the character of spacetime beyond our causal horizon.

Manchak's arguments extend prior work on the topic by making the dependence on an underdetermination argument explicit, namely through the introduction of his novel condition. He also focuses on particular kinds of underdetermining spacetimes-observationally indistinguishable spacetimes that differ in their global properties. I argue ( 3 ) that Manchak’s specific "nemesis" spacetimes fail to undermine predictions however, because they either rest on the unphysical assumption that predictions are of point-like events or are disfavored on inductive grounds. The possibility of prediction may perhaps be threatened by other underdetermining spacetimes, but not, I claim, these. I also note that his results actually do not depend on the assumption of the previously criticized conditions on prediction, and so represent a distinct thread in this literature.

If the various results proven in this literature do not undermine or pertain to prediction as I claim, what then is their significance? The most positive assessment of the consequences of the first two conditions is that they demonstrate merely that ideal observational knowledge is insufficient for making practical predictions, and we must rely on somewhat less well-justified beliefs, in addition to those given by "direct" observation, to make reliable predictions. This is, unfortunately for those who make it, not a particularly novel epistemological point, and is, in any case, already well-incorporated in standard scientific practice-as witnessed by the historical examples mentioned above and the practice of experimental gravitation. I suggest that Manchak's claims are of greater epistemological significance. They are best interpreted not in terms of prediction, since prediction applies only to global properties of spacetime in a very loose sense, but as pointing out the outstanding epistemological issue of justifying global inferences in relativistic spacetimes, an issue to which his later papers have especially drawn attention (Manchak, 2009a, 2011).

\section{Prediction: Observation and Underdetermination}

The literature on prediction in GTR employs a common strategy: explicate the concept of prediction in the standard formalism of GTR by specifying certain conditions that capture the notion of making predictions in the theory, then prove on the basis of that formalism and definitions incorporating the conditions various plausible conjectures (or provide counterexamples). The philosophical adequacy of such a method depends 
of course on whether those conditions relevantly capture the notion at issue. So, to begin, consider the following general definition utilized in this literature, adapted slightly from (Manchak, 2008, 318):

Definition 1. Given a (relativistic) spacetime and some point $q$ in that spacetime, a point $p$ is in the domain of prediction of $q$ if and only if (i) $p$ is not in the causal past of $q$ and (ii) there exists an achronal, closed, spacelike surface $S$ in the causal past of $q$ such that $p$ is in the domain of dependence of the subset $S .1$

The domain of prediction is the region of the given spacetime which is in principle "predictable" by the given observer at $q$. Two conditions are imposed on this region in the above definition, corresponding to what are taken to be intuitive limitations on the possibility of prediction for an observer (Geroch, 1977, 88-9).

Condition (i) captures the idea that predictions are not about facts in the past. As the term itself suggests, predictions are intuitively of future events. From the point of view of those advocating it, condition (i) does not really represent an important restriction however. One may define the domain of retrodiction as above but, in place of (i), with the condition that $\left(i^{\prime}\right) p$ is in the causal past of $q$. In this case, though, the domain of retrodiction is just the causal past of $q$ (Hogarth, 1997), trivially, since the observer is assumed (by all hands advocating the condition) to have in principle access to all information in her causal past $2^{2}$ This point of view is, for example, explicitly stated by Hogarth ${ }^{3}$ others appear to take the assumption as obviously valid and do not necessarily state it.

The same assumption about the powers of the observer motivates condition (ii). This condition captures the intuition that prediction is possible only when the predicted event is fully determined, and the facts that determine the predicted event are empirically accessible to the observer ${ }^{4}$ Thus the domain of dependence is the region of spacetime where events are determined from given initial data (on the achronal, closed, spacelike surface $S$ ) and a given dynamical law of temporal evolution-in the case of interest here, the Einstein field equation (EFE). The initial conditions relevant for prediction are restricted to the causal past of $q$, ostensibly on the assumption that an observer may only gather information on or from within her past light cone (Earman, 1995, 125). Note that the condition does remove all reference to the observer, but simply for the reason that "observer" is not part of the primitive language of GTR 5

On the basis of conditions (i) and (ii) alone-insofar as they are understood as explicating "the physical notion of "making predictions in general relativity'" (Manchak, 2008, 319)—one can show that prediction in GTR is subject to strict limitations, as shown by, among others, Geroch (1977) and Hogarth (1993) 6 The

\footnotetext{
${ }^{1}$ By a relativistic spacetime I mean a smooth, connected four-dimensional manifold $\mathcal{M}$ and a smooth, non-degenerate pseudoRiemannian metric of Lorentz signature $g$ defined on $\mathcal{M}$.

The timelike (respectively, causal) past of a point $q$ in a time-orientable spacetime $\mathcal{M}$ is the set of all points $p$ such that there exists a future-directed curve from $p$ to $q$ that is timelike (timelike or null). If such a curve exists, one writes $p \ll q(p<q)$. The timelike (causal) past of a point $q$ is usually denoted $I^{-}(q)\left(J^{-}(q)\right)$. The timelike (causal) future of a point $q, I^{+}(q)\left(J^{+}(q)\right)$ are defined analogously.

A closed spacelike surface $S$ in $\mathcal{M}$ is a three-dimensional subset of $M$ where every smooth curve into the subset is a spacelike curve. A subset $S$ is achronal if the intersection of $I^{+}(S)$ and $S$ is empty. The domain of dependence of a subset $S$, denoted $D(S)$, is the set of all points $p$ in $\mathcal{M}$ such that every past or future inextendible causal curve through $p$ intersects $S$.

${ }^{2}$ Of course in a relativistic spacetime not all events which fall outside an observer's past are future, since some events are spacelike related to the observer, thus one may as well allow events which are spacelike-related to the observer to count as predictable.

3"Let it be assumed that the observer at $q$ has full epistemic access to the influences in [the causal past of $q$ ]" (Hogarth 1993. 723).

4 "For present purposes take prediction to mean deterministic prediction from the laws of physics" (Earman 1995. 128).

${ }^{5}$ One may of course easily make a suitable definition. For example, "we can associate with each observer his space-time trajectory or cosmic world-line which is itself, necessarily, a future-directed timelike curve" (Malament 1977. 63).

${ }^{6}$ For example: "Given a spacetime $\mathcal{M}, g$ and a point $q$ of $\mathcal{M}$ such that [the domain of prediction of $q$ ] contains a point to the future of $q$, then $\mathcal{M}, g$ is a closed universe, in the sense that it admits a compact spacelike surface" (Geroch 1977 92). "The domain of prediction for each point in Minkowski space-time is empty. The same is true of many of the cosmological models of general relativity, e.g., the Robertson-Walker models which have been used to describe a universe beginning or ending with a big bang" Earman 1986 193).
} 
precise results are not relevant to my argument; it is sufficient to note that, as Hogarth succinctly puts it (using a similar definition of prediction), "the final word must be that the prospect of predicting the future looks pretty bleak" (Hogarth, 1993, 739).

What then of the confirmatory predictions such as of the precession of Mercury's perihelion or the bending of light? How do they fall afoul of the proofs? The answer should be evident given the definition of domain of prediction: these "predictions" are either retrodictions or more information than what is observationally accessible is taken as given in order to determine the predicted event. Set aside for the moment whether retrodictions should be thought of as predictions or not. The crucial epistemological question is, "is it justified to avail oneself of more initial data than what is observable?" That is, may an event $p$ determined from an initial data surface $S$ which is not (entirely) in the causal past of an observer be justifiably considered a prediction? If so, then one should wonder what conceptual significance the version of prediction adumbrated above is; if not, then one should be able to say why not.

The kind of argument one might give against the more permissive notion of prediction, where $S$ is not restricted to the causal past of the observer, is a sort of underdetermination argument. Such skeptical arguments take the familiar general form

1. There are some number of empirically indistinguishable $x$ 's (where $x$ may be scientific theories, spacetime models, dynamical laws, etc.).

2. The evidence cannot favor one such $x$ over any other.

3. Belief in any one of these $x$ 's is unwarranted.

Due to the demonstrable existence of observationally indistinguishable spacetimes in GTR (Glymour, 1977; Malament, 1977: Manchak 2009a), it seems on the face of it that an observer cannot know which spacetime she inhabits. If an observer relies only on evidence from her causal past, then for all she knows her spacetime could be one of any number of various empirically indistinguishable spacetimes. Her observational data therefore cannot favor one of these over any other, since none of them can be ruled out on these data alone. Therefore it seems she should conclude that she has no grounds to believe any prediction she makes based on conjectures about her spacetime outside of her causal past.

This particular underdetermination argument should in fact hardly worry the observer, though, since the dynamical laws used to determine future predictions are in this sense underdetermined as well, i.e. she cannot be certain that her prediction based on a particular dynamical law will succeed either, based solely on observations from her causal past. Insofar as one is willing to accept that GTR and its dynamical laws are adequately justified by the evidence (as conditions (i) and (ii) clearly presuppose-and as one should), the mere existence of alternate dynamical laws is insufficient to discount genuine knowledge claims deduced on their basis. Similarly, insofar as one is willing to accept that initial data which go beyond what is observable are adequately justified by the evidence, the mere existence of observationally indistinguishable spacetimes is insufficient to discount genuine knowledge claims deduced on their basis.

There are thus two problems with this naive underdetermination argument. First, it assumes that observational indistinguishability implies that evidence cannot favor one spacetime over another. It is well-known in the underdetermination literature that this is a non sequitur (Laudan and Leplin, 1991) (unless one advocates a particularly implausible and simple-minded empiricist epistemology). Second, its allowance for the justification of dynamical conditions like the EFE is in tension with the rejection of spatial conditions like an initial data surface that is not confined to the observer's causal past. The same local induction used to project the EFE into the future for the purposes of prediction is used to project conditions like homogeneity and spatial flatness outside of our causal past for the purposes of prediction 7

\footnotetext{
${ }^{7}$ Beisbart notes that common intuitions record a difference: "In physics, we distinguish between dynamical theories and the initial
} 
The failure of this argument suggests that experimental gravitation is hardly a threatened enterpriseat least its use of initial data that go beyond the restrictions of condition (ii) alone does not make it so. What then the significance of the results proved on the basis of this condition? It might be supposed the significance of condition (ii) and its consequences is primarily epistemic: predictions based on observable data are "more secure" (because better justified) than predictions based on data that go beyond an observer's causal past. The underdetermination argument from above can then be re-purposed to support this modified conclusion.

I claim that the sharp distinction between the two notions of prediction is illusory, however, for it rests on implausible assumptions about the powers of observers. It is somewhat ironic that this problem I raise comes from a failure to portray the powers of observers correctly. The various writers on this subject invariably point to the importance of distinguishing prediction and determination, precisely because prediction is an activity of observers whose ability to gather facts about their world is limited:

Questions about scientific predictability are often posed in terms of disembodied spirits whose intelligence may range over the entire spatial extent of the universe (recall Laplace's demon) or in terms of embodied observers who are given information about the past and present state of the world. But this approach leads to a never-never land form of prediction that is unavailable to actual observers who are localized and embodied and who are not "given" any free gifts of information but must ferret it out for themselves (Earman, 1986, 63).

The operative intuition behind condition (ii) is that if the putative predictable event $p$ is indeed in the domain of dependence of the initial data surface and that surface is in the causal past of $q$, then "an observer at $q$ will have at her fingertips all the information necessary to predict the state of $p$ " (Hogarth, 1993. 724). Note that she must have observed the complete surface worth of data in order to make a prediction; "missing" data correspondingly reduce the domain of dependence and therefore the domain of prediction. Obviously, observing a complete surface of data is practically impossible, so some degree of idealization is necessary. I claim however that there are not only practical impediments but properly physical impediments to an observer gathering all of the "in principle available" data, i.e. for any surface in an observer's causal past, she may only gather an incomplete set of data on that surface. This is contrary to what is assumed by all of the authors working on the topic. Hogarth, for example, intuits the observational context as follows: "Crudely speaking, [prediction] is possible because inhabitants of appropriate relativistic worlds can gather together, by physical means, all the data that determine some future event" (Hogarth, 1993, 721, emphasis added). I contend that they cannot do so, even if they tried, because well-established physics mandates that they cannot.

It is worth remarking at this point that to model observations in a spacetime theory a physical model of the phenomena by which an observer learns of her environment is required, since she cannot directly observe the purely geometrical metric or connection. Although the metric is sometimes referred to as an observable of GTR (Wald 1984, 378), this is meant in the extended sense that it is determinable by directly observable quantities (Ellis, 1980). For example, the relevant observable quantities in our universe for cosmological applications include redshifts, proper motions and angular diameter distances of astronomical objects, distortion measurements (from gravitational lensing) and number counts of distant galaxies and quasars (Ellis et al. 1985). The crucial point I wish to make, though, is that such physical models should certainly not come from the "never-never land" of physics, but should attend to how information from

conditions. The former are often supposed to hold necessarily, if they hold, whereas initial conditions are supposed to be contingent. As a consequence, induction may be on firmer grounds for laws than for initial conditions" (Beisbart 2009. 201). There is, so far as I can see, nothing decisive in these metaphysical intuitions however. 
distant regions is physically transported to the worldline of an observer-a point of which astronomers and cosmologists are obviously well aware.

Consider first an observer in Minkowski spacetime, the prototypical vacuum solution of GTR. Obviously spacetime does not have to be completely empty to be well-described by Minkowski spacetime-it is, after all, the background spacetime used in quantum field theory. The backreaction of matter and radiation on the metric only has to be negligible for it to be a good approximation of the actual spacetime. Could the observer ever gather enough data from, say, electromagnetic radiation to make a prediction by the standards of (ii)? Evidently not, since to gather enough the observer would have to receive a complete surface $S$ worth of data, and this plausibly requires photons (or whatever) communicating these data from all of $S$. But if there were a sufficient density of radiation to communicate the needed data, it would be unjustified for the observer to consider herself in Minkowski spacetime, for such a density of radiation would have a non-negligible "effect" on the metric. The assumption that motivates condition (ii) is therefore false in Minkowski spacetime and similar spacetimes, since the initial data the observer would use for determination are not accessible to the observer by any physical means.

The physics of information transportation also spells trouble for prediction in other spacetimes. Although other physical mechanisms may transport information to cosmological observers, e.g. neutrinos and gravitational waves, the principal source of information in our world is electromagnetic in nature. The familiar physics of electrodynamics by itself provides reason to doubt that an observer could ever gather a complete surface $S$ of data for the purposes of prediction from electromagnetic radiation-in any spacetime. But what is true about electrodynamics in this respect is true about other known physical theories. From what we know about physical processes (in particular on the basis of quantum field theory), information simply cannot be losslessly transported from $S$ in the past of $q$ to the observer at $q$, free of scattering, decay, or other forms of corruption.

Consider next a spacetime then where a sufficient density of information-carrying matter and radiation exist, such as the standard Friedman-Robertson-Walker spacetimes of cosmology. Is an observer in such a spacetime physically able to gather a sufficient amount of data to make predictions, as Hogarth assumes? In a transparent universe, i.e. a universe where the optical depth is everywhere zero, the answer would be affirmative. But a universe with a sufficient density of information-carrying electromagnetic radiation is not transparent, since any such radiation scatters (either through self-interactions or higher-order interactions). Thus the observed intensity of radiation emitted from a source is some fraction of the intensity of radiation emitted from that source, and the information transported via that radiation about the source is to some extent-however small—corrupted.

What this means is that for any surface of data $S$ in the observer's causal past, only a fraction of that data is accessible to the observer by physical means. But then this fraction of data is insufficient to determine events in $S$ 's domain of dependence. So the ostensibly physical assumption that motivates condition (ii) is false in any spacetime, since the initial data the observer would use for determination are not accessible to her by any physical means.

If one is indeed in the business of explicating "the physical notion of making predictions in general relativity'," then condition (ii) fails to do so, since it supposes that prediction is determination from initial data which are physically obtainable by an observer 8 No observer in any spacetime could conceivably observe a complete surface $S$ of data on physical grounds. An observer simply cannot gather the data that determine some future event by physical means. It is, in short, physically impossible.

I suspect that the motivation for condition (ii) might be defended, however, by arguing that the condition only concerns idealized observations, such that the red flags I raise over information degradation are quite besides the point. But then what precisely is the point? I have argued that the notion of an observer having

\footnotetext{
${ }^{8}$ Norton 2011) too recognizes that condition (ii) rests on "an excessively optimistic assessment of our observational abilities."
} 
complete information is physically impossible_indeed, it requires the "free gift" of information maligned above in the quotation by Earman. It seems what these authors have in mind, then, is that the proofs are meant to show that in the "best case" prediction is nearly impossible in GTR, from which one infers that in any less ideal case prediction will be correspondingly worse off. This inference, however, depends on the epistemic limitations of the ideal case carrying over to the realistic case, a point which should be resisted from a more sensible epistemological stance which accepts some degree of fallibility in our inferences.

Indeed, the foregoing considerations serve in part to emphasize that an acceptable interpretation of prediction should rely on the physically realistic assumption that an observer only has partial access to the observable data. Partial access to data is the case in practice as well as in principle; there are practical impediments as well to obtaining complete data sets, which lead to imprecise or incomplete observations. For sure, part of the power of physical theory is in using dynamical laws to determine unknown facts from a complete set of data, so partial data sets present a challenge to the application of dynamical theories. The means of overcoming this challenge are familiar though: we interpolate the data or we adopt a statistical approach. Some reasonable assumptions about the "missing" data have to be made, and so much is nothing more than standard scientific practice 9

With these remarks in mind, the seemingly innocuous condition (i) appears in a different light than that with which it was introduced previously. When observation was taken as ideal, retrodiction was trivial, so one could focus on prediction in the "strict" sense, i.e. toward the future. But partial epistemic access makes retrodiction non-trivial, and no different in kind from this strict sense of prediction (modulo concerns over entropy being relevant to the distinction between prediction and retrodiction). It therefore seems sensible to widen the scope of prediction to allow predicted events to be anywhere in the spacetime manifold at all, removing any physically or epistemically significant role for condition (i). Indeed this is the way the term "prediction" is commonly used in theoretical physics presently, and in cosmology in particular 10

In conclusion, I have argued that physical and epistemological considerations related to prediction militate against conditions (i) and (ii) as adequate or even relevant to the explication of the concept of prediction within the context of GTR. The naive underdetermination argument which one might take them as gesturing at fails in all generality, although that does not of course mean that there are not stronger underdetermination arguments that could be made. Indeed I turn to stronger such arguments in the following section, where I evaluate Manchak's contribution to the literature. But because the results are essentially based on these two conditions, they should not be interpreted in terms of prediction. Instead it seems they are perhaps better re-interpreted as something like "no-go" results on the empiricist, infallibalist epistemology that would lead to positing conditions (i) and (ii) 11 They indicate that an overly idealized conception of observation is inadequate to our predictive practices, and therefore that a realistic conception, one that acknowledges the validity of ampliative inferences, is essential to making sense of these practices.

\section{Prediction: Global Spacetime Structure}

Manchak (2008) explicitly accepts conditions (i) and (ii) as partial explications of prediction in GTR, but

\footnotetext{
9"Using observationally determined initial data, completed for those which are determined not precisely enough or which correspond to observationally not accessible regions on the past light cone, one may construct essentially local cosmological models inside the past light cone..." (Dautcourt 1983 153, emphasis added).

${ }^{10}$ Examples of this contemporary understanding of prediction abound. Here is one: "The cosmological singularity (in all examples where its character is not known to be unstable) involves infinite curvature and infinite density. One's abhorrence of such a theoretical prediction is particularly heightened by the correlative prediction that these infinities occurred at a finite proper time in the past,..." (Misner et al. 1973. 813).

${ }^{11}$ To be sure this is an epistemology that was easier to maintain in pre-relativistic days. I am inclined in fact to interpret this as the central point of (Geroch 1977).
} 
argues that they are by themselves insufficient ${ }^{12}$ His position is that an observer must not only have access to the information needed to make a desired prediction, but must also know that those facts would indeed determine the predicted state of affairs in any spacetime that is observationally indistinguishable from her own. He argues this on the grounds that her epistemic situation is such that she cannot necessarily know in which of these spacetimes she is an observer, at least given the observations she has available (Manchak 2009a). The explicit epistemic threat is that there exist spacetimes in this collection which undermine any prediction which she would like to make.

These considerations lead Manchak to define the "domain of genuine prediction" (Manchak, 2008, 320):

Definition 2. Given a spacetime and some point $q$ in that spacetime, a point $p$ is in the domain of genuine prediction of $q$ if and only if ( $i$-ii) $p$ is in the domain of prediction of $q$, and (iii), for all inextendible spacetimes, if there is an isometric embedding of q's causal past into that inextendible spacetime, then there is an isometric embedding of the union of $q$ 's causal past and p's causal past into that same spacetime such that the embeddings are equivalent when the latter is restricted to the causal past of $q .13,14$

Manchak motivates the adoption of condition (iii) by claiming that it is necessary to give "a definition of the domain of prediction which requires that the observer not only have the resources to make a prediction but also the resources to know that she can make a prediction" (Manchak, 2008, 320). He therefore finds the the definition of prediction based on only (i) and (ii) wanting, since "it seems that knowledge about one's domain of prediction requires knowledge not only of one's causal past but also of the spacetime in which one's causal past is embedded" (Manchak, 2008, 320). This is because the domain of prediction depends not only the restriction of initial data to the observer's causal past but also on the structure of the given spacetime. In this way the definition treats the structure of spacetime somewhat infelicitously as both unknown and known. In particular, it permits the assumption of global properties of spacetime, the propriety of which Manchak is keen to dispute.

In any case, condition (iii) captures the intuition that genuine prediction, i.e. prediction where the observer knows without recourse to knowledge beyond what is observationally available that the prediction is determined, requires empirically indistinguishable spacetimes to have identical observable consequences for the prediction in question. If the causal past of an observer is replicated (isometrically embedded) in another spacetime, then one can say that those spacetimes are empirically indistinguishable to that observer ${ }^{15}$ To make a genuine prediction, the observer's actual spacetime and any spacetime observationally indistinguishable from it must share the causal past of the predicted event in addition (since the causal past of the predicted event determines that event). Then she can know that her prediction is genuine, and cannot be foiled by her lack of knowledge about her actual spacetime.

Manchak (2008, 320-321) uses this novel definition to prove a theorem that depends on constructing a particular nemesis spacetime that undermines any given prediction by an observer in any given spacetime.

\footnotetext{
12 "One may wonder if the definition of the domain of prediction given above accurately reflects the physical notion of 'making predictions in general relativity.' It is [my] position that it does not" (Manchak 2008 319).

${ }^{13} \mathrm{An}$ inextendible spacetime is a spacetime for which all isometric embeddings into another spacetime are surjective. Every extendible spacetime has an (not necessarily unique) inextendible extension.

${ }^{14}$ As written, this definition is too weak. There may be multiple isometric embeddings of $q$ 's causal past into another spacetime, only one of which has the required isometric embedding of the union of $q$ 's causal past and $p$ 's causal past. In short, the observer may not only inhabit one of many observationally indistinguishable spacetimes, but may also suffer from self-locating uncertainty within a spacetime. Requiring that the first embedding is unique avoids this issue, as does requiring that the second embedding exists for each of the first.

As written, this definition is also too strong. The predicted event $p$ 's causal past need not be shared in all observationally indistinguishable spacetimes, since the entire causal past of $p$ is unnecessary to determine $p$. A suitable requirement would only have that the intersection of the future domain of dependence of the surface $S$ that determines $p$ and $p$ 's causal past is shared in all spacetimes.

${ }^{15} \mathrm{~A}$ given spacetime is completely observationally indistinguishable from another if for every point in the given spacetime, there is a point in the other where the causal pasts of the points are isometric (Malament 1977. 68). See also (Glymour 1977).
} 
He does this by making use of a global feature of spacetime-cutting a hole in one spacetime to yield another, then finding an maximal extension that preserves the hole (Manchak, 2008, 321).

Theorem 1. Given a spacetime and a point $q$, the domain of genuine prediction of $q$ is a subset of the boundary of q's causal past.

Since points that are connected to $q$ by null curves are on the boundary of $q$ 's causal past and in $q$ 's causal past, these boundary points are not in the domain of genuine prediction. Only those points that are on the boundary of $q$ 's causal past and not in the causal past are genuinely predictable according to Manchak's theorem. Thus the consequence of this theorem is that "the only possible predictions are those 'on the verge' of being retrodictions." On the basis of this theorem, he concludes that "if the epistemological predicament of the observer is fully considered, there seems to be an interesting and robust sense in which genuine prediction is not possible in general relativity" (Manchak, 2008, 321).

Manchak is careful not to run afoul of at least one of the criticisms of the previous section. He observes (in a different context, but applicably here as well) that "even under the assumption of an inductive principle — that the physical laws we determine locally are applicable throughout the universe - these general epistemological difficulties remain" (Manchak, 2011, 410). Although the quotation may suggest that he has only dynamical laws in mind, it is clear from his definition (Manchak. 2011, 413) that any local condition, such as local spatial homogeneity and isotropy, is an admissible assumption as well 16

Observe that, strictly speaking, even a single observationally indistinguishable spacetime in which the putative prediction fails is enough to preclude genuine prediction according to the definition given. This seeming demand for certainty in predictions is, as noted previously, a high standard for knowledge. It is therefore an easy target for underdetermination arguments. If the required warrant for belief is that the evidence picks out $x$ with certainty, then the mere exhibition of empirically indistinguishable $x$ 's is indeed enough to infer the conclusion of the general underdetermination argument sketched in the previous section. But if one allows that belief is justified when the warrant for belief falls short of certainty, then it is not. As noted previously, one must in that case show that the evidence cannot favor one $x$ over to some appropriate degree (Laudan and Leplin, 1991). Therefore, if the theorem is to be interpreted as demonstrating a failure of predictability, it must be because this nemesis spacetime is not disfavored by evidence compared to the actual spacetime.

Before turning to evaluate Manchak's theorem, I note that my remaining criticisms are in fact irrelevant to it. Although Manchak incorporates condition (ii) into his definition of genuine prediction, his theorem does not in fact depend at all on its presence. The definition of genuine prediction is easy to modify:

Definition 3. Given a spacetime and some point $q$ in that spacetime, a point $p$ is in the domain of genuine prediction of $q$ if and only if ( $\left.i i^{\prime}\right)$ there exists an achronal, closed, spacelike surface $S$ such that $p$ is in the domain of dependence of the subset $S$, and (iii'), for all inextendible spacetimes, if there is an isometric embedding of the union of $q$ 's causal past and $S$ into that inextendible spacetime, then there is an isometric embedding of the union of q's causal past and p's causal past into that same spacetime such that the embeddings are equivalent when the latter is restricted to the union of the causal past of $q$ and $S$.

Manchak's theorem is provable on the basis of this definition without any significant modification of the details of the proof.

\footnotetext{
${ }^{16}$ As Norton comments, "Our observable spacetime is four-dimensional and has a Lorentz signature metrical structure. We are allowed the inductive inference that this will persist in the unobserved part. More generally, we are allowed to infer inductively to the persistence of any local condition, such as the obtaining of the Einstein gravitational field equations, in both the observer's and the [observationally indistinguishable] spacetimes" (Norton 2011 170).
} 
The theorem and its proof - the latter of which makes essential use of global features of spacetime-are motivated by the thought that there is a significant epistemic difference between extending conditions locally and extending them to all of spacetime. Manchak for example suggests that "induction on such large scales would seem to be suspect, given that we are able to observe only a 'negligibly small region' of the universe" (Manchak, 2011, 416). Even if we were able to observe a large fraction of the universe, though, one might argue that such local observations could still never justify belief in a global property of spacetime. Indeed, in keeping with this latter sentiment, (Manchak, 2009a) argues that there is a "robust sense in which the global structure of every cosmological model is underdetermined" by observationally indistinguishable spacetimes.

It is important to note, though, that Manchak first proposes a different nemesis spacetime than the one constructed in the theorem, one in which the predicted event's spacetime point is simply removed from the observer's manifold. He does not dwell on this simple failure of predictability however. This mutilated spacetime is extendible (just add the point back), and "one could require that spacetime be inextendible" (Manchak, 2008, 320). Since requiring spacetimes to be such is common in the relativity literature, he requires that potential nemesis spacetimes be inextendible in his definition of genuine prediction. For precisely the reasons given in the previous paragraph one may wonder whether the global property of inextendibility is in fact epistemically justified. Indeed, Manchak (2011) himself argues that it is sometimes problematic to insist on inextendibility, since not every well-behaved spacetime has a well-behaved maximal extension and not every spacetime has a unique extension.

Nevertheless, Manchak's theorem proves that even under the assumption of inextendibility any observer's spacetime is underdetermined in a way that undermines any given prediction she wishes to make. Absent an argument to favor the observer's spacetime, it seems that there are defeasible reasons to take the underdetermination seriously and conclude that genuine prediction is essentially impossible. The observer, after all, cannot tell the difference between the two spacetimes based on her observations. On the other hand, one might wish to see an argument that the nemesis' spacetime and the actual spacetime are equally warranted for the underdetermination threat to withstand scrutiny.

Manchak (2011) provides some support for the latter claim, arguing that a compelling case for a categorical prohibition of the kind of spacetime holes used to construct the nemesis spacetime has not been made ${ }^{17}$ While the global property of hole-freeness is often assumed to hold by fiat in practice, Earman (1995, 98) diagnoses the motivation for holding it as a mere desire to preserve determinism, an attitude he argues is impermissible and question begging when determinism is at issue ${ }^{18}$

I certainly have no argument as to whether hole-freeness is a physically motivated condition or not. I am therefore happy to agree with Manchak's arguments which conclude that hole-freeness is an unjustified assumption. Nevertheless I do claim that the specific nemesis spacetime in Manchak's theorem is not a threat to making predictions in GTR. Indeed, an observer in that spacetime would consider it predictively indistinguishable with respect to the spacetime from which it is constructed, and so the theorem cannot be proved on its basis.

Recall that a fundamental assumption of relativistic spacetime theory is that spacetime is a continuum and appropriately modeled by a manifold. It is usually assumed that every point in the spacetime manifold represents an event. These assumptions, however, are on somewhat dubious empirical grounds, since no measurement could possibly ever register any such pointlike event. Observable events are in point of fact always larger than a point. (It is of course usually convenient to treat them as points in most formal mod-

\footnotetext{
${ }^{17}$ In support of the physical reasonableness of spacetime holes, he cites Geroch (1971b 78): "The space-times obtained by cutting and patching are not normally considered as serious models of our universe. However, the mere existence of a space-time having certain global features suggests that there are many models—some perhaps quite reasonable physically—with similar properties."

${ }^{18}$ Manchak 2009b) agrees, and for this reason adopts the view that it may be possible to demonstrate hole-freeness in GTR by assuming suitably physically motivated conditions.
} 
els.) Since predictions are of measurable events, we should therefore demand that any theorem that treats predicted events as pointlike must show that that prediction is sufficiently "robust" in order to have physical significance ${ }^{19}$

The construction of the nemesis spacetime in Manchak's theorem begins by cutting out the boundary of a closed spacelike surface of the actual spacetime (the boundary including the pointlike prediction), this surface itself in some (open) neighborhood of the pointlike prediction. Cutting such a hole, however, does not affect the properties of the remaining points in this neighborhood. (One stitches two of these "holed" spacetimes together to make an inextendible spacetime-Manchak does not demonstrate how they are patched together, but it can be shown.) Thus it cannot be truly said that these two spacetimes differ in their authentic predictions, since the actual prediction an observer makes is more robust than a single missing point, line, or surface-the predicted event is intuitively an open region. Observers in the two spacetimes would in fact make identical authentic predictions (up to an arbitrary degree of measurement accuracy). Thus, with a suitably modified condition (iii), Manchak's theorem cannot be proven for such physically significant predictions using this particular nemesis spacetime.

One might think that the modification to recover the result is trivial, since it seems that larger holes could easily be cut out. That is, for any open region $O$ one requires in order to insure robustness of predictions, remove a closed region including the open region $O$ needed to insure the robustness of predictions, such that one can then follow Manchak's recipe for patching together copies of that spacetime. Such a procedure will not work, however, insofar as one maintains the requirement of inextendibility. The spacetime constructed in this way can always be extended to the nemesis spacetime constructed in Manchak's original proof (just replace all the points save one, keeping the existence of a hole intact), which spacetime, as argued above, fails to invalidate the observer's prediction.

Perhaps some different inextendible nemesis spacetime can be constructed that meets the "robustness of predictions" requirement I introduce and also invalidates any observer's predictions. It may even be of some interest to investigate this possibility, but as it is a crucial epistemological issue remains so long as one does not produce grounds to justify inextendibility. Manchak's initial nemesis spacetime, where one takes the actual spacetime and removes the predicted point, can easily be adapted however to invalidate predictions no matter how robust one demands them to be. That is, without the inextendibility requirement, one can cut as big of a hole as one requires. Thus the real issue that needs resolution is whether such "holed" spacetimes are disfavored over their "unholed" counterparts.

Again, I have no desire to argue for or against hole-freeness as a physical constraint on spacetime. For my purposes I need only claim that the nemesis spacetime which involves removing predictions by cutting holes should be disfavored in order to rebut Manchak's claim. I argue for this also on inductive grounds. In our causal past we have never observed any physical consequence of such an arbitrary hole (for example indeterministic evolution which would best be explained by the presence of such a hole), and for this reason we have good reason to expect that our predictions will not be prevented by such arbitrary holes in our future in the way that Manchak suggests. Any observer who has not observed such physical consequences in her spacetime would reasonably make the same inference. (There may yet have been holes in our causal past which have had no observable consequences, but the existence of these holes at least would not bolster any argument against disfavoring prediction-undermining holes in our future.)

I conclude therefore that the prediction of events in spacetime is not at all essentially impossible —at least for the reasons that Manchak produces. Introducing spacetime holes in the way of the nemesis spacetimes merely represents a kind of restricted skepticism about prediction that can be resisted on straightforward inductive and physical grounds as I have argued. If one extends the scope of prediction to global properties

\footnotetext{
19“"But if a prediction depends crucially upon the precise data-if it undergoes a drastic change under even arbitrarily small perturbations of that data — then our prediction, while perhaps suggestive and useful, has little physical significance" (Geroch 1971a).
} 
of spacetime, then it can perhaps be said that our ability to predict is limited (Manchak, 2009a), but this usage only loosely makes contact with the practically relevant notion of making and confirming predictions in GTR. Nonetheless, I emphasize that the underdetermination of global spacetime structure represents a peculiar and interesting epistemological challenge that has not yet been decided; it is, however, a challenge which is only misleadingly described as a challenge for prediction.

\section{Concluding Remarks}

Geroch (1977) proposes that a discussion of predictions in physics only makes sense in the context of a particular physical theory. He remarks that therefore "one might divide a discussion of prediction in physics into two parts: (1) the choice of a physical theory and (2) the establishment and interpretation of certain theorems within the mathematical formalism of that theory" (Geroch, 1977, 81). Such a method is fine as far as it goes ${ }^{20}$ if one's aim is merely to gain some insight into a theory or to seek modifications to a theory that has certain pathologies, then there is much to recommend such a strategy in the philosophy and foundations of physics.

The physical and philosophical significance of the results of an investigation conducted in this manner can be easily distorted however-precisely because the explication of a concept in a theory depends sometimes sensitively on physical details and philosophical motivations. For example, when discussing the prospects of prediction in Newtonian mechanics, where the possibility of "space invaders" (particles entering or leaving spacetime from "infinity") ruins the uniqueness of solutions to an initial value problem, Geroch suggests fixing this theoretical flaw by requiring that no information comes into spacetime from infinity. He remarks, however, that "this result would not, at least to me, suggest prediction, for it constrains both the initial state of the system and its future behavior" (Geroch, 1977, 82). Yet surely dynamical laws constrain a system's future behavior as well. The apparently diverging epistemic standards Geroch thus holds for dynamical constraints and non-dynamical constraints is, I suppose, a philosophical predilection; rejecting this distinction, a distinction which is in any case epistemologically ill-founded, would straightforwardly obviate the suppositious claim that "there seems to be no theorem in ordinary Newtonian mechanics that suggest possibilities for prediction" (Geroch, 1977, 82).

I have argued that claims of the impossibility of prediction in the general theory of relativity are similarly suppositious, and unsoundly so. As they are interpretations of technical results in terms of the concept of prediction, they depend on the availability of precise and relevant explications of this concept. I have argued that the conditions which constitute these explications are ill-motivated and defective. Conditions (i) and (ii) are unphysical and over-idealized in a way that greatly limits their significance; condition (iii) is problematic as well, as it fails to recognize the physical import of robustness. If one is to mount a compelling argument that a technical result pertains to prediction, one requires better conditions than these. One must, that is, pay close attention to the philosophical considerations, especially if the concept to be explicated is an essentially epistemological one like prediction.

I also argued that Manchak's nemesis spacetimes constitute insufficient grounds for a compelling underdetermination argument for undermining the possibility of predictions. My arguments parallel standard moves for disputing underdetermination arguments: a putative case of underdetermination is argued either to fail to be distinguished in the relevant way or it is disfavored on epistemological grounds. In one case this is because the spacetime does not yield a different prediction, and in the other inductive arguments straightforwardly favor the unmutilated spacetime, at least in worlds like our own.

\footnotetext{
${ }^{20}$ Geroch, at least, acknowledges that to follow this method is to operate in a "rather narrow framework" (Geroch 1977. 83).
} 
Insofar as prediction is an activity of "actual observers who are localized and embodied and who are not 'given' any free gifts of information," I conclude that one should not be led to motivate these conditions nor maintain that prediction is in any sense impossible because of results proved on their basis. There remains one question: "What then is the significance of the theorems proved?"

I urged that Manchak's argument is best understood as focusing attention on an important and underinvestigated epistemic challenge, namely the justification of assuming global properties of spacetime. Only in a loose sense of prediction can these properties be thought of as predictable; nonetheless, the arguments justifying such assumptions leave much to be desired, a contention which Manchak has emphasized elsewhere (Manchak, 2011).

The arguments based on consequences of conditions (i) and (ii) however appear to serve only as pointing out that idealized observations and naive deduction are insufficient to predict anything in GTR. Earman at least appears to take this to be a significant epistemological point, remarking that "observation combined with inductive reasoning may recommend one hypothesis over all others, but rarely, if ever, does the combination yield a confidence that approaches the certainty with which Laplace's demon went about its prediction tasks." (Earman, 1986, 66). Perhaps some still cling to bygone fancies of certitude in our epistemological endeavors, in which case the point may still resound; for the most part that philosophy and science have long since come to terms with the fallibility of knowledge, indeed it seems without any apparent forfeiture of confidence in the practical prospects of prediction.

\section{References}

Beisbart, Claus. "Can We Justifiably Assume the Cosmological Principle in Order to Break Model Underdetermination in Cosmology?" Journal for General Philosophy of Science 40: (2009) 175-205.

Dautcourt, Georg. "The cosmological problem as initial value problem on the observer's past light cone: geometry." Journal of Physics A: Mathematical and Theoretical 16: (1983) 3507-3528.

Earman, John. A Primer on Determinism. Dordrecht, Netherlands: D. Reidel Publishing Company, 1986. Bangs, Crunches, Whimpers, and Shrieks. Oxford: Oxford University Press, 1995.

Ellis, George. "Limits to Verification in Cosmology." Annals of the New York Academy of Science 336: (1980) 130-160.

Ellis, George, S. Nel, Roy Maartens, William Stoeger, and Andrew Whitman. "Ideal Observational Cosmology." Physics Reports 124: (1985) 315-417.

Geroch, Robert. "General Relativity in the Large.” General Relativity and Gravitation 2: (1971a) 61-74.

"Spacetime Structure from a Global Viewpoint." In General Relativity and Cosmology, edited by Rainer Sachs, New York: Academic Press, 1971b.

- "Prediction in General Relativity." In Foundations of Space-Time Theories, edited by John Earman, Clark Glymour, and John Stachel, Minneapolis: University of Minnesotra Press, 1977, volume 8 of Minnesota Studies in the Philosophy of Science, 81-93.

Glymour, Clark. "Indistinguishable Space-Times and the Fundamental Group." In Foundations of SpaceTime Theories, Minneapolis: University of Minnesota Press, 1977, volume 8 of Minnesota Studies in the Philosophy of Science, 50-60. 
Hogarth, Mark. "Predicting the Future in Spacetimes." Studies in History and Philosophy of Science 24: (1993) 721-739.

- "A Remark Concerning Prediction and Spacetime Singularities." Studies in History and Philosophy of Modern Physics 28: (1997) 63-71.

Laudan, Larry, and Jarrett Leplin. "Empirical Equivalence and Underdetermination." The Journal of Philosophy 88: (1991) 449-472.

Malament, David. "Observationally Indistinguishable Space-Times." In Foundations of Space-Time Theories, edited by John Earman, Clark Glymour, and John Stachel, Minneapolis: University of Minnesotra Press, 1977, volume 8 of Minnesota Studies in the Philosophy of Science, 61-80.

Manchak, John. "Is Prediction Possible in General Relativity?" Foundations of Physics 38: (2008) 317-321.

- "Can we know the global structure of spacetime?" Studies in History and Philosophy of Modern Physics 40: (2009a) 53-56.

—. "Is spacetime hole-free?" General Relativity and Gravitation 41: (2009b) 1639-1643.

__. "What Is a Physically Reasonable Space-Time?" Philosophy of Science 78: (2011) 410-420.

Misner, Charles, Kip Thorne, and John Wheeler. Gravitation. San Francisco: W. H. Freeman, 1973.

Norton, John. "Observationally Indistinguishable Spacetimes: A Challenge for Any Inductivist." In Philosophy of Science Matters: The Philosophy of Peter Achinstein, edited by Gregory Morton, Oxford: Oxford University Press, 2011, chapter 13, 164-176.

Wald, Robert. General Relativity. Chicago: University of Chicago Press, 1984.

Will, Clifford. "The Confrontation between General Relativity and Experiment." Living Reviews in Relativity 9: (2006) 1-100. [Online Article]: cited [27 Jan 2013], http://www.livingreviews.org/lrr-2006-3. 